\title{
Rediscovering FISH in the midst of a sperm chromatin conundrum
}

\section{David F. Albertini ${ }^{1}$}

Published online: 17 October 2019

(C) Springer Science+Business Media, LLC, part of Springer Nature 2019

As much as we reach for solutions to the ART dilemma of selecting "good stuff," the natural world's predilection for shades of gray continues to stymie our hopes for a yes/no (sic binary) type of answer. Take for instance the question I often receive from patients and practitioners alike regarding how to choose the best oocyte. The answer is I have no clue. This response is all that can be mustered despite the fact that for many experienced oologists, including authors of wellknown atlases of human oocyte morphology, sorting out the grossest of dysmorphisms has done little to improve our ability to choose the best oocyte in any way that might predictably aid in determining outcomes of import. In the end, what our selection strategies do is offer a best guess.

Extending this mindset to embryos, and now evermore sperm chosen for ICSI, has become a mainstay for contemporary ARTs. This month we take our readership on a journey through the perplexing world of sperm selection seen through and beyond the lens of chromatin organization. We start with the newer application of a seasoned technology, that of fluorescence in situ hybridization, or FISH. For those of you who have a desire to actually see what you are talking about, let us not forget that FISH may have had a checkered past with respect to the early days of PGD/PGS but years later, it remains an evolving and highly adaptable method for understanding chromatin organization at a level not easily deduced by the latest and greatest bioinformatics-based methods like aCGH or NGS. Whether the upgrades made in this technology will serve to better understand the heterogeneity and complexity of sperm populations for clinical utility is the subject of the paper by Sarrate and colleagues (The use of fluorescence in situ hybridization analysis on sperm: indications to perform and assisted reproduction technology outcomes, https://doi. org/10.1007/s10815-019-01554).

David F. Albertini

eicjarg@gmail.com

1 Center for Human Reproduction, New York, NY, USA
In the context of aneuploidy within sperm populations, many studies have now elaborated on the need to better understand genetic factors that may impact male infertility directly [1] or those that may reside within the context of how genome organization itself influences either sperm viability or most importantly what the consequences of using genetically compromised sperm would be in terms of clinical outcomes especially after ICSI. Towards this end, Tempest and colleagues have been probing the matter of three-dimensional chromatin organization as a potential cause for infertility [2]. In addition, interesting and important questions remain with respect to the mechanisms underlying aneuploidy during meiosis and the ability of chromosomally imbalanced gametes to escape what were thought to be rigorous cell cycle checkpoints that should have eliminated aneuploid sperm within the testis [3]. Seems now that besides genetic determinants gone awry, we will have to consider in more detail the spectrum of epigenetic and seminal fluid influences at work in the landscape of male infertility!

Beyond the level of detecting sperm aneuploidies and working to understand their role in normal reproduction and ARTs, paternal aging and the impact of the environment continue to attract attention given the evidence showing decreased fertility in many male populations around the world. There is now good evidence to support an environmental impact [4]. And adding to the conundrum at hand is the growing body of literature identifying epigenetic disturbances prior to or during lifespan of males that play out as transgenerational patterns of inheritance appearing in offspring from exposed individuals [5].

This month's issue contains a number of papers that bridge the subject of sperm quality from the point of view of paternal age (Advanced paternal age does not affect embryo aneuploidy following blastocyst biopsy in egg donor cycles, https://doi. org/10.1007/s10815-019-01549), semen quality and embryo mosaicism (Male factor infertility impacts the rate of mosaic blastocysts in cycles of preimplantation genetic testing for aneuploidy, https://doi.org/10.1007/s10815-019-01584), and the introduction of microfluidic devices for sperm sorting ( $A$ 
treatment approach for couples with disrupted sperm DNA integrity and recurrent ART failure, https://doi.org/10.1007/ s10815-019-01543). While each of these studies portends current and future problems begging for more research, we should keep in mind that our current approaches to sperm selection may themselves have an influence on chromatin integrity as evidenced by the use of chromatin fragmentation assays [6]. Much debate continues with regard to the specificity, sensitivity, and meaning of chromatin fragmentation assays performed on heterogeneous sperm samples with or without selection.

Our issue this month covers many other subjects ranging from the latest advances in human IVM, to the costs of hospitalization in ART cycles, and novel systems for extending the reach of fertility preservation. We hope you enjoy our offerings this month as well as the upcoming ASRM meeting in Philadelphia. We would love to hear your ideas and suggestions and hope you will consider stopping by the Springer/ Nature booth (\#2007) in the exhibit hall.

\section{References}

1. García-Mengual E, Triviño JC, Sáez-Cuevas A, Bataller J, RuízJorro M, Vendrell X. Male infertility: establishing sperm aneuploidy thresholds in the laboratory. J Assist Reprod Genet. 2019;36(3):37181. https://doi.org/10.1007/s10815-018-1385-0.
2. Ioannou D, Tempest HG. Does genome organization matter in spermatozoa? A refined hypothesis to awaken the silent vessel. Syst Biol Reprod Med. 2018;2:1-17.

3. Ioannou D, Fortun J, Tempest HG. Meiotic nondisjunction and sperm aneuploidy in humans. Reproduction. 2019;157:R15-31.

4. Perry MJ, Young HA, Grandjean P, Halling J, Petersen MS, Martenies SE, et al. Sperm aneuploidy in faroese men with lifetime exposure to dichlorodiphenyldichloroethylene (p,p-DDE)and polychlorinated biphenyl (PCB) pollutants. Environ Health Perspect. 2016;124:951-6. https://doi.org/10.1289/ehp.1509779.

5. Dickson DA, Paulus JK, Mensah V, Lem J, Saavedra-Rodriguez L, Gentry A, et al. Reduced levels of miRNAs 449 and 34 in sperm of mice and men exposed to early life stress. Transl Psychiatry. 2018;8: 101. https://doi.org/10.1038/s41398-018-0146.

6. Muratori M, Tarozzi N, Carpentiero F, Danti S, Perrone FM, CambiF $\mathrm{CA}$, et al. Sperm selection with density gradient centrifugation and swimup: effect on DNA fragmentation in viable spermatozoa. Sci Rep. 2019;9:7492. https://doi.org/10.1038/s41598-019-43981.

Publisher's note Springer Nature remains neutral with regard to jurisdictional claims in published maps and institutional affiliations. 